\title{
POTENCIAL CLIMÁTICO PARA A CULTURA DO DENDÊ NA MESORREGIÃO DE RIBEIRÃO PRETO-SP
}

\author{
VASCONCELOS, Ricardo de Lima ${ }^{1}$ \\ GÍACOMO, Karina dos Santos ${ }^{2}$ \\ SILVA, Leandro Aparecido Alves ${ }^{3}$ \\ GARCIA, Anice ${ }^{4}$
}

Recebido em: 2009.12.19

Aprovado em: $2010-01.25$

ISSUE DOI: $10.3738 / 1982.2278-354$

RESUMO: O objetivo deste trabalho foi verificar se a mesorregião de Ribeirão Preto-SP apresenta potencial agroclimático para a cultura do Dendê. $\mathrm{O}$ estudo se baseou em dados de precipitação e temperatura médias mensais de sete cidades do estado de São Paulo (Barretos, Bebedouro, Cristais Paulista, Guaíra, Ituverava, Morro Agudo e Ribeirão Preto). O balanço hídrico climatológico foi elaborado pelo método Thornthwaite ; Mather (1955) através do programa BH norm em planilha EXCEL® por Rolim et al. (1998). Os limites climáticos para determinação do potencial climático para o Dendê foram adaptados de Cassiano (2008), SEAGRI (2009), Souza (2009), MAPA (2009), Caramori (2006), Embrapa (2009), Duke (2009), Macedo Junior et al. (2009) e Bastos (2001) citando Moraes; Bastos (1972). Pelos resultados obtidos observou-se que as cidades analisadas, representativas da mesorregião de Ribeirão Preto-SP mostraram-se restritas para a cultura do Dendê, necessitando de uma irrigação complementar.

Palavras-chave: Balanço hídrico climatológico. Bioenergia. Elaeis guineensis Jacq

\section{CLIMATE POTENTIAL FOR OIL PALM CULTURE IN MESOREGION OF RIBEIRÃO PRETO-SP}

SUMMARY: The purpose of this work was to evaluate the agroclimatic potencial for oil Palm culture in mesoregion of Ribeirão Preto- SP. The study bases on the pluvial precipitation data and on the monthly mean temperatures of seven sites of state São Paulo (Barretos, Bebedouro, Cristais Paulista, Guaíra, Ituverava, Morro Agudo e Ribeirão Preto). The climatological water balance was developed by the Thornthwaite ; Mather (1955) method trough the BHnorm program in EXCEL $®$ charts; by Rolim et al. (1998). Climatic limits to determine the potential climate for the oil Palm were adapted from Cassiano (2008), SEAGRI (2009), Souza (2009), MAPA (2009), Caramori (2006), Embrapa (2009), Duke (2009), Macedo Junior et al. (2009) and Bastos (2001) citing Moraes; Bastos (1972). The results obtained showed that the cities examined, representing the mesoregion of Ribeirão Preto-SP were more restricted to the culture of oil Palm, requiring supplementary irrigation.

Keywords: Climatic water balance. Bioenergy. Elaeis guineensis Jacq .

\footnotetext{
${ }^{1}$ Engenheiro Agrônomo. Faculdade Dr. Francisco Maeda/FAFRAM, 14500-000, Ituverava/SP - e-mail: ricardovasconcelosagro@yahoo.com.br

${ }^{2}$ Mestrando, Faculdade Dr. Francisco Maeda/FAFRAM, 14500-000, Ituverava/SP - e-mail: karinagiacomo@hotmail.com

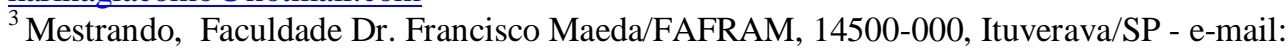

alves10leandro@yahoo.com.br

${ }^{4}$ Engenheira Agrônoma, Professora Doutora em Agrometeorologia da Faculdade Dr. Francisco

Maeda/FAFRAM, 14500-000, Ituverava/SP - e-mail: anice@ feituverava.com.br
} 


\section{INTRODUÇÃO}

Miragaya (2005) enfatiza que disponibilidade de fontes agrícolas para produção do biodiesel varia de acordo com o clima e as condições das regiões de produção, e o Brasil apresenta reais condições de se tornar um dos maiores produtores de biodiesel do mundo. A cultura do dendezeiro (Elaeis guineensis Jacq) fornece um óleo, que se destaca por ser o segundo mais produzido no mundo, oferecendo uma grande gama de exploração, é responsável por $80 \%$ da produção mundial de margarina, gorduras sólidas, óleo de cozinha, maionese, panificação, leite, chocolate artificial e também é usado como matéria-prima para diferentes segmentos nas indústrias óleo-químicas, farmacêuticas, de sabões e cosméticos (EMBRAPA 2002a). O Brasil e, particularmente a Amazônia apresentam um grande potencial para a expansão do cultivo de dendê e a cultura pode ser considerada como mais uma opção para recuperar áreas degradadas (SANTOS et al., 2005).

Os mesmos autores comentam que o estado do Pará é o maior produtor de dendê do país, que em 1998 registrou uma área colhida de 14.339 hectares, com uma produção de 145.785 toneladas, correspondendo a um rendimento médio de 10,17 toneladas de cachos de frutos/ha. No ano de 2003, a área colhida salta para 44.463 ha e uma produtividade de 16,4 toneladas/ha, o que demonstra um avanço na pesquisa para elevar o crescimento da atividade na região.

Para Beltrão (2005) o dendezeiro no Brasil pode produzir 35 bilhões de litros de óleo, que através da transesterificação, resultaria em igual quantidade de biodiesel, sendo portanto superior a produção da Arábia Saudita em derivados de petróleo em um ano, o dendezeiro começa a produzir a partir do terceiro ano após o plantio e sua produção varia de 4 a 6 toneladas de óleo/há ${ }^{-1}$ ano, distribuída ao longo do ano, por mais de 25 anos consecutivos. Por ser uma cultura perene, utiliza intensiva de mão-de-obra, sem entressafras, permite a fixação do homem no campo, em média, cada 10 hectares de dendezal, garante trabalho rentável para uma família (EMBRAPA, 2002b). Além disso, em termos ecológicos ambientais apresenta uma contribuição significativa, capaz de contribuir com o seqüestro de 29,3t de carbono/ $\mathrm{ha}^{-1}$ ano na fase adulta (SOUZA, 2000).

A distribuição mensal da chuva e a ocorrência de déficit hídrico são os elementos climáticos que apresentam maior efeito no crescimento e na produção da do dendê, e que além de um total pluviométrico anual acima de $1.500 \mathrm{~mm}$, uma boa distribuição mensal e ausência de período seco, proporcionam ambiente climático ideal para a cultura e totais de chuva entre' 120 mm e 150 mm, constituem limite mínimo de chuva mensal satisfatório para a produção do dendezeiro e que quanto menor é o déficit hídrico, maior é a produção anual de 
cachos (MORAES ; BASTOS, 1972; OCHS ; DANIEL, 1976; FERWERDA, 1977; HARTLEY, 1977; BARCELOS et al., 1987; MULLER ; ALVES, 1997; BASTOS, 2000; CARVALHO, 2000 citados por BASTOS (2001).

Bastos (2001) comenta ainda a influência da deficiência hídrica na masculinização dos cachos e conseqüentemente redução na produção de frutos, inviabilizando a exploração comercial do óleo, sendo para esta cultura um ótimo ambiente de produção requer precipitações anuais acima de $1500 \mathrm{~mm}$; as precipitações mensais devem estar em torno 120 mm (AGRIANUAL, 2008), porém as precipitações reportadas como ideais, em que há maiores produções são de $2500 \mathrm{~mm}$ anuais e $150 \mathrm{~mm}$ mensais (EMBRAPA, 2002c).

Temperaturas médias entre 24 a $28^{\circ} \mathrm{C}$ são consideradas como ideais (EMBRAPA, 2002c), sendo que o limite máximo e mínimo de temperatura varia de 28 a $34^{\circ} \mathrm{C}$ e 21 a $23^{\circ} \mathrm{C}$ e que a deficiência hídrica anual deve ser menor que $100 \mathrm{~mm}$ para o bom desenvolvimento do cultivo (BASTOS, 2001). Duke (2009) observa que em temperaturas abaixo de $15^{\circ} \mathrm{C}$ há uma redução da germinação de sementes. Teixeira (2005), reforça a importância da temperatura, pois baixas temperaturas ocasionam a solidificação do óleo, inviabilizando seu uso na região sul do país, o biodiesel do dendê é um subproduto industrial, seu custo estaria próximo ao do biodiesel de sebo bovino. O dendezeiro é a planta que apresenta a maior produtividade de óleo por área cultivada, produzindo, em média, 10 vezes mais óleo do que a soja, em condições ecológicas mais favoráveis, produz 8 toneladas de óleo por hectare/ano (EMBRAPA, 2002c).

Por ser uma cultura ainda não é explorada no estado de São Paulo, mas com promissoras chances de crescimento na área de bioenergia, o objetivo de desse trabalho é verificar se mesorregião de Ribeirão Preto-SP apresenta potencial climático para a cultura do Dendê (Elaeis guineensis Jacq).

\section{MATERIAL E MÉTODOS}

O presente estudo se baseou em dados médios anuais de precipitação e temperatura do ar, de sete municípios pertencentes a mesorregião de Ribeirão Preto-SP. Os dados de precipitação e temperatura do ar para a cidade de Ituverava-SP $\left(20^{\circ} 20^{\prime} 22^{\prime \prime} \mathrm{S}, 4^{\circ} 46^{\prime} 50^{\prime \prime} \mathrm{W}\right.$ e 605 m), cujo clima de acordo com a classificação de Köppen, é Aw, com verão úmido e inverno seco (CARRER ; GARCIA, 2007), foram obtidos na Estação Agrometeorológica da Faculdade Dr. Francisco Maeda (FAFRAM). Para a cidade de Morro Agudo (2043'53" S, 48 $03^{\prime} 28^{\prime \prime}$ W e $546 \mathrm{~m}$ ), os dados foram fornecidos pela Companhia Açucareira Vale do Rosário. Para a cidade de Ribeirão Preto-SP ( $21^{\circ} 10^{\prime} 39^{\prime \prime}$ S, 4748’37" W e 546 m), os dados 
foram fornecidos pela estação experimental do Instituto Agronômico. Para as cidades de Barretos-SP (20³3'26" S, 48 34'04" W e 530 m), Bebedouro-SP (2056'58" S, 48²8'45" W e 573 m), Cristais Paulista-SP (20²3'50" S, 47²5'13" W e 996 m ), Guaíra-SP (20¹9'06" S, 48 18'38" W e $517 \mathrm{~m}$ ) os dados foram obtidos pelo Centro Integrado de Informações Agrometeorológicas (CIIAGRO). Esses dados foram utilizados na elaboração do balanço hídrico climatológico, empregando-se o método de Thornthwaite ; Mather (1955), através do programa BHnorm elaborado em planilha EXCEL® por Rolim et al. (1998).

A capacidade de água disponível (CAD) utilizada para a confecção dos Balanços Hídricos foi de $200 \mathrm{~mm}$. Como resultado, o balanço hídrico forneceu as estimativas da evapotranspiração real (ETr), da deficiência hídrica (DEF), do excedente hídrico (EXC) e do armazenamento de água no solo (ARM) para cada mês do ano. Os registros de dados foram criteriosamente analisados, de forma que os valores duvidosos de precipitação, falhas de preenchimento e outras situações foram descartadas e corrigidas.

Os limites climáticos para determinação do potencial climático para o Dendê foram adaptados de Cassiano (2008), SEAGRI (2009), Souza (2009), MAPA (2009), Caramori (2006), Embrapa (2009), Duke (2009), Macedo Junior et al, (2009) e Bastos (2001) citando Moraes; Bastos (1972) como mostra a Tabela 1.

Tabela 1. Faixas de classificação dos parâmetros utilizados e aptidão de risco climático do potencial do Dendê.

\begin{tabular}{lcc}
\hline Classes & Precipitação $(\mathbf{m m})$ & Temperatura $\left({ }^{\circ} \mathbf{C}\right)$ \\
\hline Viável & $1200-2000$ & $19-32$ \\
\hline Ideal & $1800-2500$ & $21-28$ \\
\hline Restrita por Def. Hídrica & $<1200$ e $<100 \mathrm{~mm} / \mathrm{mes}$ & $19-32$ \\
\hline Restrita por Def. Térmica & $1200-2.500$ & $<19$ \\
\hline Inviável & $\begin{array}{c}250 \text { mm def. anual e/ou }+2 \text { meses } \\
\text { deficiência hídrica } \geq 50 \mathrm{~mm}\end{array}$ & $\begin{array}{c}\text { reduz germinação } \\
<15\end{array}$ \\
\hline
\end{tabular}

\section{RESULTADOS E DISCUSSÃO}

São apresentados na Tabela 2, os valores de temperatura média do mês mais quente, temperatura média do mês mais frio, precipitação média anual e deficiência média anual baseada em séries históricas dos sete municípios estudados. 
Tabela 2. Valores médios de temperatura do mês mais quente (Tq), do mês mais frio (Tf), precipitação média anual $(\mathrm{P})$ e deficiência média anual (DEF) para os municípios estudados.

\begin{tabular}{lccccc}
\hline Município & Período & Tq $\left({ }^{\circ} \mathbf{C}\right)$ & Tf $\left({ }^{\circ} \mathbf{C}\right)$ & P $(\mathbf{m m})$ & DEF $(\mathbf{m m})$ \\
\hline Barretos & $1992-2008$ & 26,7 & 20,7 & 1439,7 & 138,8 \\
\hline Bebedouro & $1992-2008$ & 25,7 & 20,4 & 1385,4 & 117,1 \\
\hline Cristais Paulista & $1992-2008$ & 24,0 & 18,7 & 1495,1 & 56,8 \\
\hline Guaíra & $1991-2008$ & 26,0 & 20,3 & 1589,6 & 85,2 \\
\hline Ituverava & $1996-2008$ & 25,5 & 19,5 & 1460,1 & 127,7 \\
\hline Morro Agudo & $1985-2005$ & 26,4 & 19,9 & 1516,6 & 107,0 \\
\hline Ribeirão Preto & $1976-2006$ & 24,6 & 19,4 & 1470,3 & 72,5 \\
\hline
\end{tabular}

A análise do parâmetro temperatura média demonstra que todas as cidades avaliadas não apresentam restrição quanto à temperatura para o cultivo de Dendê, pois a variação da temperatura média do mês mais quente $(\mathrm{Tq})$ foi de $24,0^{\circ} \mathrm{C}$ (Cristais Paulista) a $26,7^{\circ} \mathrm{C}$ (Barretos), valores que se enquadram dentro dos limites exigidos para o cultivo de Dendê que é de 21 a $28{ }^{\circ} \mathrm{C}$; e em relação a temperatura média do mês mais frio, a menor temperatura foi a apresentada por Cristais Paulista $\left(18,7{ }^{\circ} \mathrm{C}\right)$ evidenciado uma restrição térmica.

Quanto ao parâmetro precipitação, não há restrição por parte das cidades que apresentam valores acima dos requeridos pela cultura, mesmo as menores precipitações que foram encontradas para a cidade de Bebedouro (1385,4mm), são maiores que limite inferior da classe da viabilidade de cultivo.

Na Figura 1 são apresentados os extratos do Balanço Hídrico Climatológico, nos quais é possível observar os períodos de deficiência hídrica, excedente hídrico, retirada hídrica e reposição hídrica, para cada município estudado.

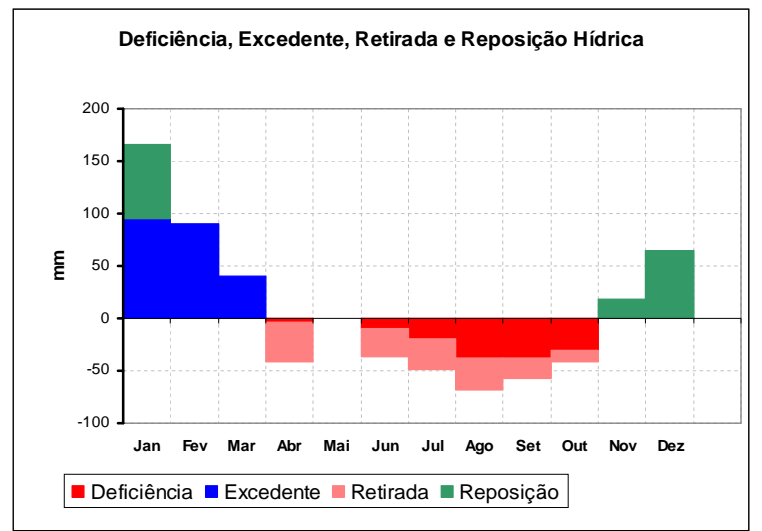

Barretos-SP

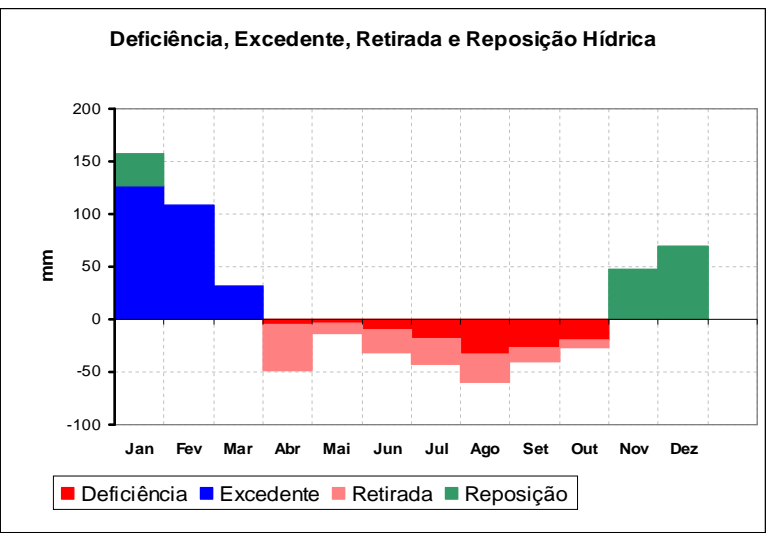

Bebedouro-SP 


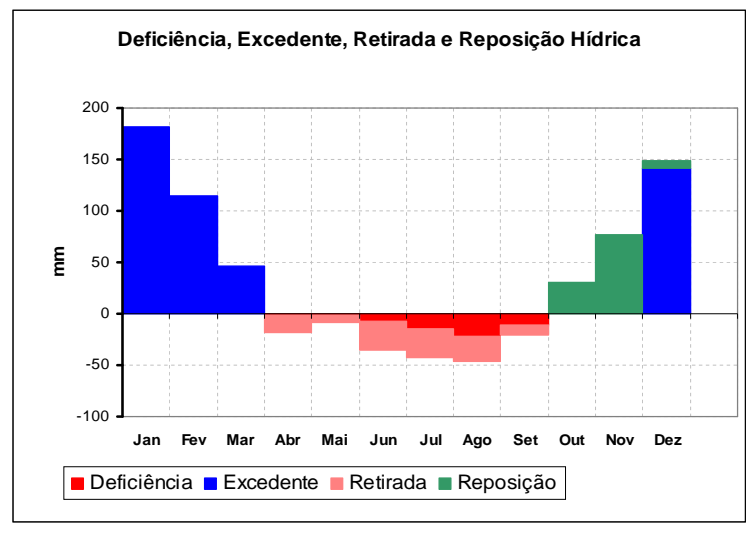

Cristais Paulista-SP

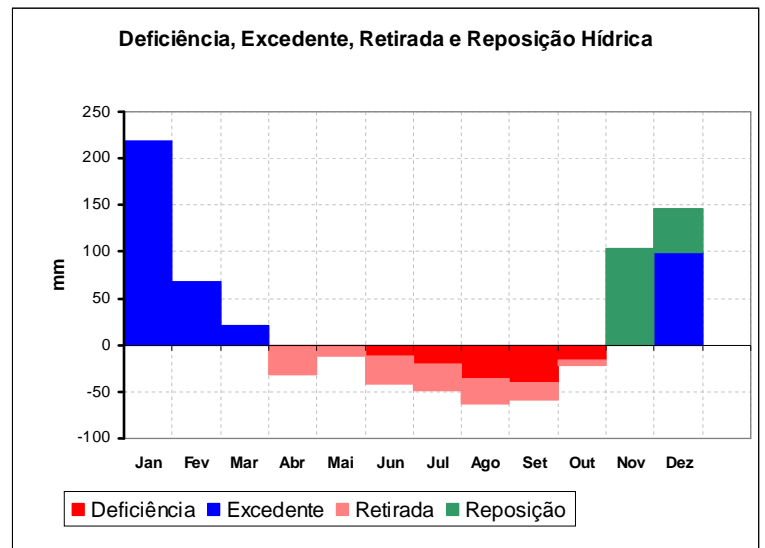

Ituverava-SP

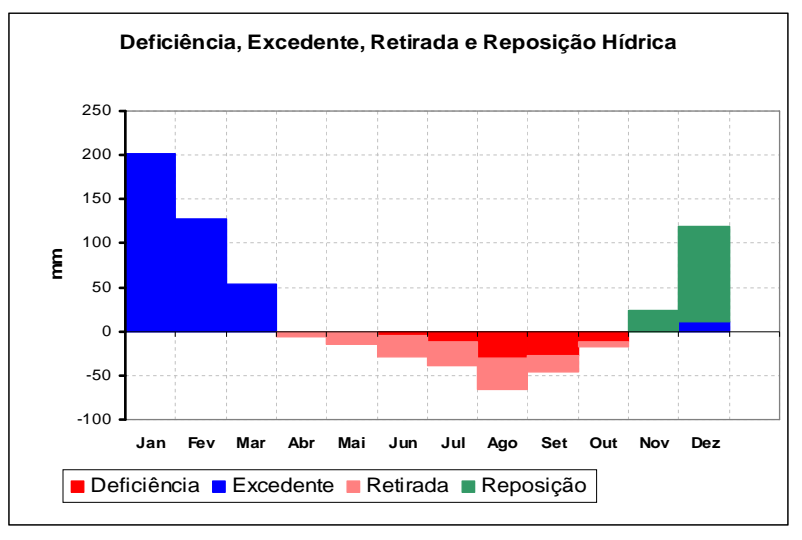

Guaíra-SP

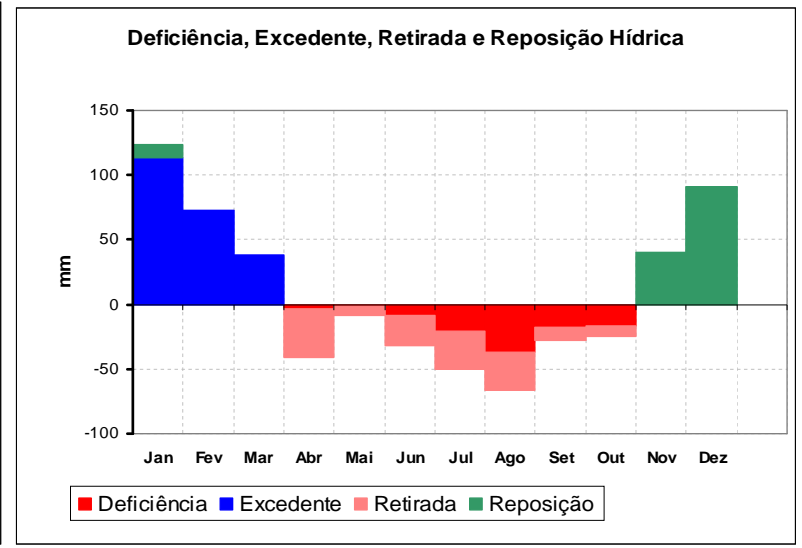

Morro Agudo-SP

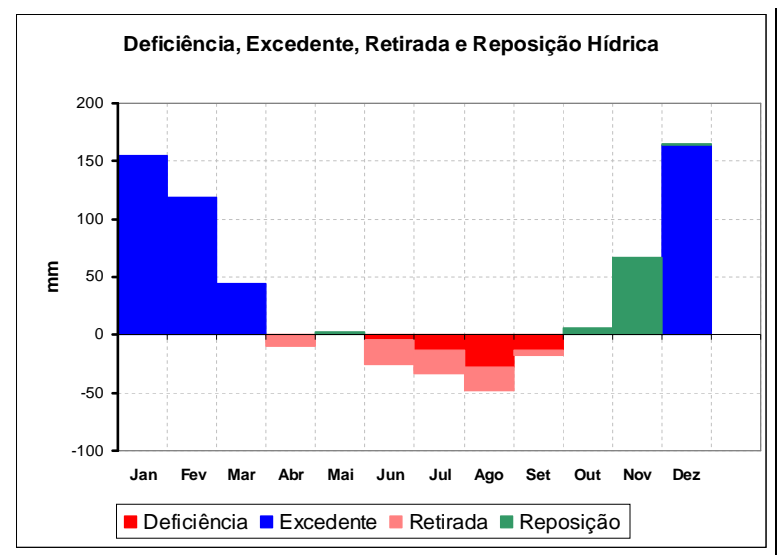

Ribeirão Preto-SP

Figura 1. Extratos dos Balanços Hídricos Climatológicos para cada uma das cidades estudadas, baseados em séries históricas.

Com relação aos períodos de excedentes hídricos, eles variam de janeiro a março para as cidades de Barretos, Bebedouro e Morro Agudo, e de dezembro a março para as demais localidades. 
Já pelos períodos de deficiência hídrica, observa-se que, com exceção das cidades de Cristais Paulista e Ribeirão Preto, que apresentam o período de deficiência hídrica de junho a setembro e Guairá, que apresenta período de deficiência de julho a outubro, para as demais cidades esse período se estende de junho a outubro.

A deficiência hídrica média anual das cidades estudadas variou de 138,8mm para a cidade de Barretos e 56,8mm para a cidade de Cristais Paulista. Mas quando se verifica o período de deficiência hídrica mensal, encontram-se dois grupos distintos de cidades, aquelas em que se registrou apenas um ou nenhum mês de deficiência hídrica igual ou superior a 50 mm (Cristais Paulista, Ribeirão Preto, Bebedouro, Morro Agudo e Guaíra) e as cidades de Barretos e Ituverava que registraram dois meses consecutivos de deficiência hídrica superior a $50 \mathrm{~mm}$.

Entretanto, a precipitação média mensal, durante os meses de abril a setembro, em todas as localidades foi inferior a $100 \mathrm{~mm}$, valor abaixo do qual é considerado restritivo à cultura.

\section{CONCLUSÃO}

A partir das informações obtidas e da literatura consultada, as cidades estudadas apresentam-se como restritas à cultura uma vez que, embora as temperaturas médias e a precipitação anual atendam as necessidades da cultura, a distribuição irregular (precipitações menores que $100 \mathrm{~mm}$ em alguns meses) torna a região restrita à produção comercial, sem uma complementação de irrigação.

\section{REFERÊNCIAS}

AGRIANUAL. Anuário da agricultura brasileira. São Paulo: FNP, 2008. p. 46-47.

BASTOS, T. X. et al. Zoneamento de riscos climáticos para a cultura do dendezeiro no estado do Pará. Revista Brasileira de Agrometeorologia. Passo Fundo, v.9, n.3, 2001. p.564-570.

BELTRÃO, N. E. M. Agronegócio das oleaginosas no Brasil. In: Informe Agropecuário: produção de oleaginosas para o biodiesel. Belo Horizonte: EPAMIG. v.26, n.229, p. 14-17. 2005.

CARAMORI, P.H. Zoneamento agroclimático das principais plantas oleaginosas do Brasil. In: SIMPÓSIO DO AGRONEGÓCIO DE PLANTAS OLEAGINOSAS, 2. Anais... Piracicaba, 2006.

CARRER, T.T.; GARCIA, A. Classificação climática para a cidade de Ituverava/SP. Nucleus, Ituverava, v.4, n. 1-2, p. 39-47, 2007. ISSN 1678-6602.

CASSIANO, W. Uma nova visão da Amazônia. AgRIANUAL 2008. São Paulo: FNP. p. 4647. 
CIIAGRO. Centro Integrado de Informações Agrometeorológicas. Disponível em: http://www.ciiagro.sp.gov.br/. Acesso em: 18 out. 2009.

DUKE, J. A. Handbook of energy crops. Disponível em:

www.hort.pordue.ed/newcrop/duke_energy/ Elaeis_guineensis.html. Acesso em: 28out.2009.

EMBRAPA - Empresa Brasileira de Pesquisa Agropecuária. O que é Dendê? Disponível em: http://www.cpaa.embrapa.br/portfolio/sistemadeproducao/dende/dendeoquee.htm.

EMBRAPA-Amazônia Oriental, 2009a. Acesso em:13 set. 2009.

EMBRAPA- Empresa Brasileira de Pesquisa Agropecuária. Dendê: Alternativa de desenvolvimento sustentável para agricultura familiar na Amazônia brasileira. Disponível em: http://www.cpaa.embrapa.br/servicos/livraria/arquivos_gratis/folderdendejackson.pdf. EMBRAPA-Amazônia Oriental, 2009b. Acesso em: 13 set. 2009.

EMBRAPA - Empresa Brasileira de Pesquisa Agropecuária.. Onde plantar dendê no Brasil. Disponível em:

http://www.cpaa.embrapa.br/portfolio/sistemadeproducao/dende/dendeondeplantar.html. 2002c. Acesso em 13 set. 2009.

MACEDO JUNIOR, C. ASSAD, E. D; MARIN, F. R. Zoneamento de riscos climáticos para a dendeicultura no Brasil. In: CONGRESSO BRASILEIRO DE AGROMETEOROLOGIA 16, 2009, Belo Horizonte, MG. Anais...Belo Horizonte, 2009.

MAPA - Ministério da Agricultura, Pecuária e Abastecimento. Disponível em: http://extranet.agricultura.gov.br/sislegis-consulta/servlet/VisualizarAnexo?id=15668 . Acesso em: 28 out. 2009.

MIRAGAYA, J. C. G. Biodiesel: tendência no mundo e no Brasil. Informe Agropecuário: produção de oleaginosas para o biodiesel. Belo Horizonte: EPAMIG. v.26, n.229, p.7-13. 2008.

SANTOS, M. A. S; FILGUEIRAS, G. C; PINHEIRO, M. S. A. Avaliação das fontes de crescimento da dendeicultura no estado do Pará no período 1990-2003. In: CONGRESSO DA SOCIEDADE BRASILEIRA DE ECONOMIA E SOCIOLOGIA RURAL 43. Anais... Ribeirão Preto, 2005.

SEAGRI. Secretaria da Agricultura, Irrigação e Reforma Agrária. Disponível em: C:IDocuments and SettingsłpesquisalConfigurações locais\TempไDiretório temporário 5 para dende.zip\SEAGRI - Cultura Dende.mht . Acessado em: 29 out. 2009.

SOUZA, J. Dendê. Disponível em: C:\Documents and Settings|UsuáriolConfigurações locais\Temp\Diretório temporário 2 para dende.zip\Dend+ᄀ.mht. Acessado em 28 out.2009.

ROLIM, G.S; SENTELHAS, P.C; BARBIERI, V. Planilhas no ambiente EXCEL para os cálculos de balanços hídricos: normal, sequencial, de cultura e de produtividade real e potencial. Revista Brasileira de Agrometeorologia, Santa Maria, v.6, p.133-137, 1998.

TEIXEIRA, L. C. Potencialidades de oleaginosas para a produção de biodiesel. Informe Agropecuário: produção de oleaginosas para o biodiesel. Belo Horizonte: EPAMIG. v.26, n.229, p. 18-27. 2008.

THORNTHWAITE, C.W; MATHER, J.R. The water balance. Centerton, NJ: Drexel Institute of Technology - Laboratory of Climatology, Publications in Climatology, v. 8, n.1, 104p., 1955. 